\author{
Proceedings of the $9^{\text {th }}$ International Conference on Applied Informatics \\ Eger, Hungary, January 29-February 1, 2014. Vol. 1. pp. 295-304 \\ doi: $10.14794 /$ ICAI.9.2014.1.295
}

\title{
Application of machine vision in manufacturing of bearings using ANN and SVM
}

\author{
Krisztián Deák, Imre Kocsis, Attila Vámosi \\ University of Debrecen Faculty of Engineering \\ deak.krisztian@eng.unideb.hu, kocsisi@eng.unideb.hu, \\ vamosi.attila@eng.unideb.hu
}

\begin{abstract}
Bearings have a vital role in nearly all rotating machines. Making adequate bearings is very important that satisfy all needs which emerge both in manufacturing and during operation. In former times bearings were examined by only humans, however human inspection is instable and time consuming. In this article, we are investigating a machine learning system that could make more accurate measurements regarding geometry, shape, color, surface defects, deformations and other failures by image acquisition. To achive higher resolution, magnifying of the surface with optical microscopes and scanning electron microscope (SEM) is inevitable. With these methods even tiny failures can be detected. Machine learning methods have beeen developed such as artificial neural networks (ANN) and support vector machines (SVM). Bearing manufacturing failures, image processing techniques are presented in this article besides artificial neural network system that can percieve manufacturing defects approximately $90 \%$ efficiency according to our experiments. Recent research is connected to a manufacturing of bearings in a real company in Hungary.
\end{abstract}

Keywords: machine vision, bearings, manufacturing, ANN, SVM

\section{Introduction}

Bearings are central parts of nearly all machines in automotive industry and manufacturing machines. It is very important to achive outstanding manufacturing quality and to reduce harmful factors that influence the products and the process themselves. Parameters such as metallographic material structure and the geometry of the bearing parts, waviness, surface roughness are seriously regulated and should be taken into consideration in manufacture. 
Machine learning methods and image processing help to decide manufacturing and operation problems together with image processing and signal processing based on time domain analysis and frequency domain analysis. Basically, artificial neural networks (ANN), support vector machines (SVM), hybrid systems (e.g. ANFIS), fuzzy approaches can give efficient tool for analysing bearing defects and other machine defects. Recently, it is a trend to substitute human inspection and apply automatic methods to enhance the efficiency of the expert systems.

\section{Bearing types and manufacturing process}

Roller bearings have basic parts, two rings that reinforce the set of elements running between the rings on the raceways. Furtehr elements are inner ring, outer ring, cage, sealing that prevent contaminations to get to the bearing. Lot of types are defined such as cylindrical roller, tapered roller, needle, and barrel roller bearings. Ball bearings are classified in three categories: radial, thrust, and angularcontact. Deep-groove bearings are the most widely used ball bearings that can carry substantial thrust loads at high speeds in either direction. Internal and external self-aligning bearings are distinguished coming in two types: internal and external. Figure 1. shows a possible application of tapered roller bearings and its basic parts. They are frequently used in automotive gear boxes.
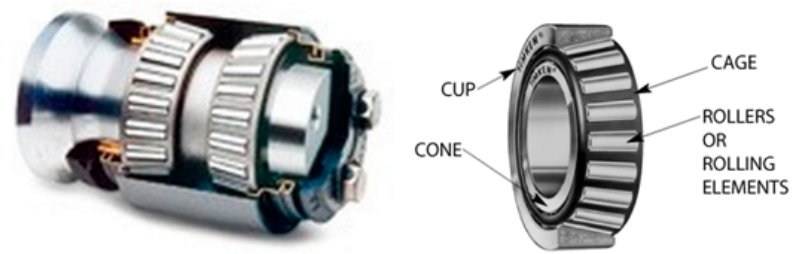

Figure 1: Tapered roller bearing application (left) and its parts

(right) [3]

Several bearing defects emerge in production, here a short description of the steps of the manufacture that are in connection with the later mentioned waviness, surface roughness, metallographic failures. Metal balls are manufactured from soft wire with a certain diameter. Wire is cut in smaller sections and its diameter become nearly equal to its length. In this phase it has spherical shape. Hardened steel rill plates de-flashe the wire. Basically, one of these plates is stationary the other is in motion. The top plate has an opening to allow balls to enter and exit the rill plates. These plates have fine circumferential grooves that the balls track in. The balls go through the machine which ensures each ball is the same size, even if a particular groove is out of specification.[1] Balls need grinding in different steps. If the balls are steel they are heat treated. After heat treatment they are descaled to remove any residue or by-products.[1] Then, the balls are hard grounded. Next, smoothing is applied to make the surface of the balls even and reduce the value 
of the roughness parameteres. They are grounded in the same type of machine as used before, but either an abrasive is introduced into coolant or the rotating plate is replaced with a very hard fine-grain grinding wheel. This step can get the balls within $\pm 0.0025 \mathrm{~mm}$ accuracy. If the balls need more precision then they are lapped, again in the same type of machine, finally superfinishing is applied to maked the final surface quality. Rings are manufactured in the same way, they are machined with turning and grinding machines, hardened, tempered, washed and cooled. Raceways are perfectly grinded and superfinished according to the product requirements. Cage is made from steel plate by cutting sections. Finally bearing elements are assemblied, filled with grease or lubricants, sealed and wrapped.

Waviness and surface roughness are critical factors and largely depends on the manufacture process. Wavy raceways can result in localized and distributed faults, increased friction, wear, noise, vibration, heat and $\mathrm{CO}_{2}$ emission which are very strictly regulated by all countries in the world. That is why very important to put emphasize on this issue and take it seriously.

\section{Image processing in detection of bearing defects}

Geometric measurings of bearings and bearing parts are constantly improved in bearing manufacture. Sophisticated equipment with diverse measuring devices for dimensional and form/ shape inspection are applied both on the spot in the quality assurance and in laboratories. It is very important to inspect the following parameters with different image processing techniques that are used in this bearing manufacture company which uses very strict regulations therefore they can produce bearings of excellent quality. [17]

- Length and diameter measurement by micrometers.

- Inspection of dents, cracks, scratches on the surfaces. (Fig. 3.)

- Roughness profile (Fig. 4.) measurements down to one hundredth of a micrometer.

- Deviation of roundness check with up to 100000 fold magnitude including frequency analysis of waviness. (Fig. 5.)

- Inspection of shape (Fig. 6.) and radius with a magnification of up to 100 000 fold.

- Inspection of bearing clearances and radial runout of individual parts.

- Inspection of form and position tolerances on form measuring systems and coordinate measuring machines, also for very irregularly formed construction parts such as cast steel housings.

$100 \mathrm{Cr} 6$ bearing material is usually applied for bearings that consists of: C $0.93 \%-1.05 \%$, Si $0.15-0.35 \%$, Mn $0.25-0.45 \%$, Cr $1.35-1.60 \%$. During 

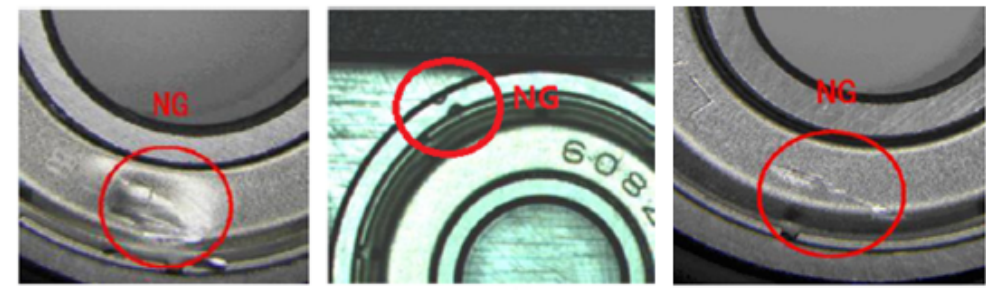

Figure 2: Basic bearing faults (dents, scratches) due to possible manufacturing problems
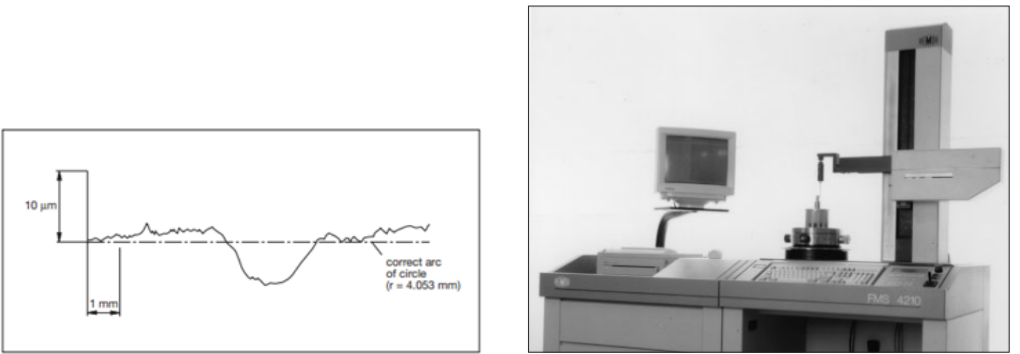

Figure 3: Profile measurement (left) and shape measurement (right) of a ball bearing elements[17]
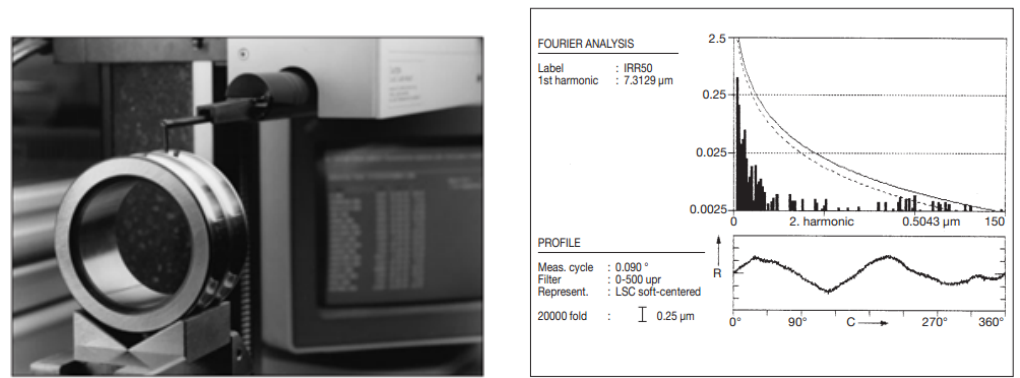

Figure 4: Inner ring waviness measurement and Fourier analysis of waviness [17]

manufacture several changes in the material content could occur that influence the mechanical properties of the material under certain loads and operation conditions. Heat treatment alters the structure of the steel. Heat treatment temperature and time are important factors, too. Because of quality assurance reasons constant destructive and non-destructive tests are required, as see below:

- Metallographic assessment of structure by optical microscope or SEM (Fig. 5.)

- Making zones of unpermissible heating visible by etching the contact areas 
- Crack inspection by means of ultra-sound or eddy current besides image analysis

- Radioscopic measuring of retained austenite in the material

- Inspection of material cleanliness by optical microscope or SEM
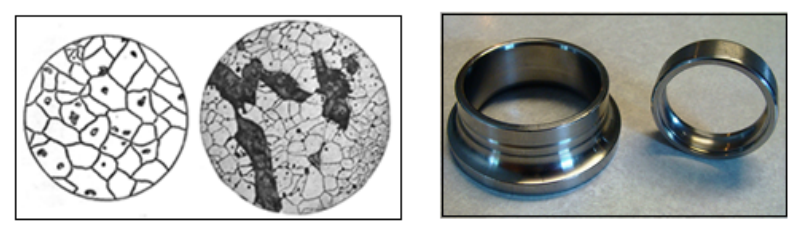

Figure 5: Metallographic analysis (left) and heat affacted zone analysis (right) [5]

Machine learning methods in manufacturing Lot of defects in manufacture could emerge that badly influence the quality of the bearings. Human inspection is even widespread in bearing companies but it has disadvantages such as time consuming method and unreliable because decision depends on human factors. By using machine learning like artifical neurals networks (ANNs) [19], support vector machines (SVMs) [16] the reliability, accuracy, precision and efficiency of the inspection can be increased. Figure 6. presents the structure of ANN and the hyperplane of SVM.

Beyond ANNs and SVMs, Fuzzy approaches [14], hybrid systems, like Adaptive Neuro-Fuzzy Inference System (ANFIS) [11, 4], multi-layer feed-forward [5], radial basis function [8], wavelet neural networks [13], adaptive resonance theory networks are widely applied. Patter recognition models [15], automated fuzzy inference [10] and genetic algorithms [9] are further applied to assist automatic bearing inspection.
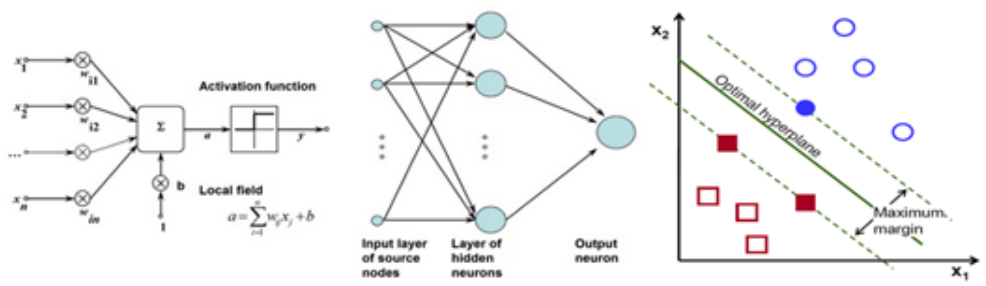

Figure 6: Artifical Neural Network schematic (left, middle) and SVM (rigth) [19, 13]

SVM estimates the connection between predictive variables and explanatory variables. Maximal margin approach and kernel method are combined in SVM to make prediction. Support Vector Machine (SVM) is a classification and regression method. Support Vector Machine (SVM) is a state-of-the-art method, frequently 
used as nonlinear classifier or learning algorithm which is able to evaluate automatically dependency between data and defined as a regression problem. Support Vector machines uses hypothesis space of linear functions in a high dimensional feature space. Separation is achieved by the best hyperplane that has the largest distance to the nearest training data point of any class, the functional margin, the distance from the hyperplanes to the nearest data point on each side is maximized. SVM decision function is an application of the kernel function and Lagrangian optimization method is used to obtain the optimal decision function from the training data. $[18,16]$

\section{Experiments and tests for detecting metallograph- ic, surface texture and geometrical faults}

Metallographic study the structure of the materials was achieved by optical microscopes (LOM) and electron microscopes (SEM or TEM) in this experiment (Fig. 7., 8.) This method is applied is bearing production as well and necessary before image acquisition to make visible the phase boundaries. Without preparation of the specimen analysis is impossible.

$100 \mathrm{Cr} 6$ steel is generally used in bearing manufacture that has martensitic structure with a large amount of retained austenite (RA\%) along the grain boundaries. This steel has high fatigue strength and hardness, and good wear resistance. RA\% was measured in the experiments by image software of the Olympus BX61 microscope under 100X and 500X.

Firstly, a section of the steel was cut from the raw material before any treatment. Secondly, materials after heat treatment were analysed and the change in their material structure is estimated. Sections are called specimens that are bedded in resin with hot temperature under high pressure. (Fig. 8.) Hot compression thermosetting resins fix the metal then it can be polished and etched before microscope investigation. 3\% Nital was used for etching which is a solution of alcohol and nitric acid. Alcohol is ethanol or other methylated liquids. This preparation was excellent to enhance the material phase boundaries. Prepared specimens were examined with the unaided eye after etching to detect any visible areas that have responded to the etchant differently from the norm as a guide to where microscopical examination should be employed. Low magnification rates below 500X produce better image contrast so software based digital image enhancement not always necessary. SEM and TEM can produce magnification rates up to 3000X or even more. Metallographic measurement usually determines the volume fraction of a constituent or a phase or measures the grain size in polycrystalline metals and alloys. Furthermore, size, shape, form and distribution of particles, spacing between the particles are also measured. Here, remained austenite phase value was measured which is good indicator for supervising the technological process.

As the part of metallographic investigation digital image processing methods were applied to obtain the information necessary to make further decision. Here, 
in this article and experiments LOM and SEM miscoscopes were used to the 2D image acquisition. Then, it was necessary to remove background noise by filtering in order to assure that sensor noise does not introduce false information. Geometrical transformations such as rotating were done. Then images were pre-processed to extract some specific piece of information. Contrast enhancement was applied to assure that relevant information can be detected. Feature extraction is the extraction of image features at various levels of complexity. Edge detection was used to detect edges of the material phases, contaminations in the $100 \mathrm{Cr} 6$ steel. Prewitt edge detector was used and compared to Sobel detector that produced higher efficiency. Thresholding was applied to set and determine the gray value percentage of the LOM and SEM images. Segmentation helped to divide the digital image into multiple segments to simplify and/or change the representation of an image into something that is more meaningful and easier to analyze. Image manipulations were made with ImageJ and MATLAB using its Image Processing Toolbox. (Fig. 8.)

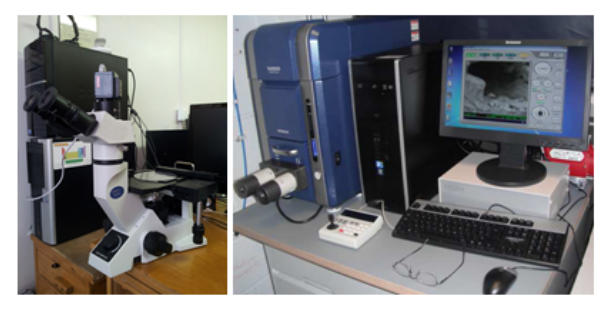

Figure 7: Olympos BX61 optical microscope with analytic software (left) and HITACHI TM 3030 SEM (right)
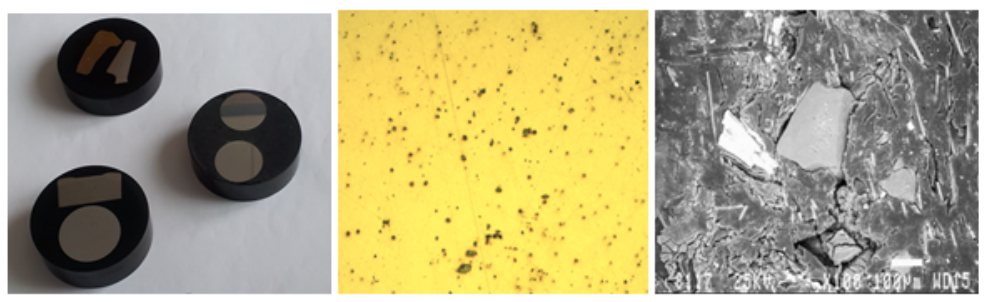

Figure 8: Metallographic specimens and analysis with the optical microscope (left), after image processing for RA\% calculation (middle), and SEM (right)

For surface geometrical measurements waviness analysis was applied which is the measurement of the more widely spaced component of surface texture. The next reasons contribute for waviness fault: machine or work deflections, chatter, residual stress, vibrations, or heat treatment, wearing of manufacturing machines, machine vibration, excentric motion of the workpieces, excessive tool wear. Wa and $\mathrm{Wt}$, for average waviness and total waviness, respectively.[6] In the lateral direction 
along the surface, the waviness spacing, Wsm, is another parameter that describes the mean spacing between periodic waviness peaks. Waviness is included in the ISO standards ISO 4287[6] and ISO 16610-21[7] as well as the U.S. standard ASME B46.1.[2]. Mahr Perthometer (Fig. 9.) was used its plastic tip was connected to an electronic pickup which then measured the surface variations. Furthermore, in bearing manufacture, surface roughness is usually measured, which is a measure of the texture of a surface.

Beyond traditional $\mathrm{R}_{a}, \mathrm{R}_{z}, \mathrm{R}_{q}, \mathrm{R}_{p}, \mathrm{R}_{c}, \mathrm{R}_{v}, \mathrm{R}_{s k}, \mathrm{R}_{k u}$ (ISO 4287) in bearing manufacture special bearing waviness indicators are measured such as $\mathrm{R}_{k}$, the Core Roughness Along (X, Y) parameters, are derived from the bearing ratio curve based on the ISO 13565-2:1996 standard. For each profile, a bearing area curve is generated by simulating a horizontal line moving through the profile from the top down, evaluating the percentage of contact the line would make with the surface at each level. $\mathrm{R}_{p k}$, the Reduced Peak Height Along $(\mathrm{X}, \mathrm{Y})$, is found from a measure of the peak height above the core roughness. $\mathrm{R}_{v k}$, the Reduced Valley Depths Along (X, $\mathrm{Y}$ ), is found from a measure of the valley depths below the core roughness. $\mathrm{R}_{k}$ is a measure of the core (peak to valley) roughness of the surface with the major peaks and valleys High $\mathrm{R}_{p k}$ implies a surface composed of high peaks providing small initial contact area and thus high areas of contact stress when the surface is contacted. $\mathrm{R}_{p k}$ may represent the nominal height of the material that may be removed during a running-in operation. $\mathrm{R}_{v k}$ is a measure of the valley depths below the core roughness and may be related to lubricant retention and debris entrapment.[12] In this experiments these parameters were measured but were not processed for machine learning application they would be the part of further experiment.
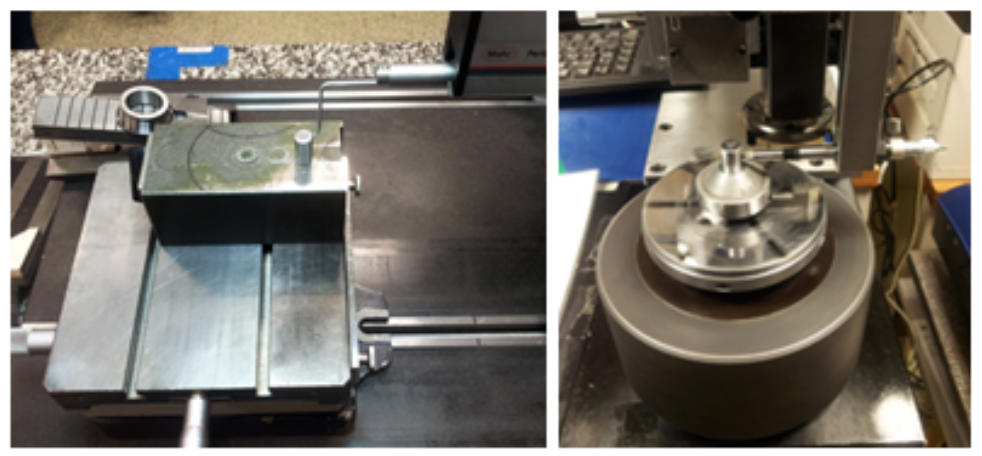

Figure 9: Roller shape measurement and waviness measurement during manufacture

\section{Artificial Neural Network for classifying bearing maufacture defects}

In this experiment we focused on automatic machine learning application in order to avert uncertainty of human inspection. Artificial Neural Network (ANN) was built 
in MATLAB using Neural Network Toolbox. Levenberg-Marquart backpropagation algorithm as an alternative of Newtoin gradiens was used because it gives results within a short time. ANN has four input parameters, one metallographic RA\% and three waviness parameters Wa, Wt, Wsm which are good indicators both the material structure and the shape/ form requirements of the bearing rings and rolling elements. The network has two outputs: material defect and waviness defect. Efficiency of the ANN reached $88 \%$ for material defects and $93 \%$ for waviness defects.

\section{Conclusion}

Bearings are critical and important machine elements in nearly all machines. It is necessary to produce high quality products that meet the strict requirements. In this article, a possible machine learning application was presented in the bearing manufacture industry. Lot of standards give instructions how to make bearings, now we focused on the metallographical analysis of the material and the measurement of geometrical parameters of the bearing rings and rolling elements. All of them are essential factors to satisfy the manufacture requriments and to ensure long lifetime later on. Optical and electrone microscope were used for surface texture investigation. Image processing techniques were applied to help the analysis. Waveometer was applied for waviness measurement. Artificial Neural Networks was built in MATLAB with four inputs and two outputs using Levenberg-Marquart backpropagation algorithm. High efficiency was reached but further aim is to enhance the efficiency of material defect recognition. Using more sophisticated image processing techniques it might be possible. Further researches are planned to use SVMs, surface roughness parameters besides the waviness parameters. Later, we would like to reveal the possible deeper connections between the surface parameters of manufacture defects and noise/ vibration acceleration values.

\section{References}

[1] Abbottball Company: Bearing maunufacture process. http://www . abbottball.com/ about-abbott/today/manufacturing.php

[2] ASME B46.1 Standard, Surface Texture (Surface Roughness, Waviness, and Lay).

[3] Bright Hub Engineering portal: http://www.brighthubengineering.com/ machine-design/26455-types-of-bearing-ball-bearings /

[4] Ghafari S.H., Golnaraghi F., and Ismail F., "Rolling element bearings fault diagnosis based on neuro-fuzzy inference system," Proceeding of CMVA 2006

[5] He Z., Wu M., and Gong B., "Neural network and its application on machinery fault diagnosis," Proceeding of IEEE International Conference on Systems Engineering, 1992, pp. 576-579.

[6] ISO 4287 Standard, Geometrical Product Specifications (GPS) - Surface texture: Profile method Terms, definitions and surface texture parameters 
[7] ISO 16610 Standard, Geometrical product specifications (GPS) - Filtration - Part 21: Linear profile filters: Gaussian filters

[8] Jack L.B., Nandi A.K., and McCormick A.C., "Diagnosis of rolling element bearing faults using radial basis function networks," Applied Signal Processing , v 6, 1999, pp. 25-32.

[9] Lei Y., He Z., Zi Y., and Hu Q., "Fault diagnosis of rotating machinery based on multiple ANFIS combination with GAs," Mechanical Systems and Signal Processing, 2007, pp. 2280-2294.

[10] Liu T.I., Singonahalli J.H., and Iyer N.R., "Detection of Roller bearing defects using expert system and fuzzy logic," Mechanical Systems and Signal Processing, v 10, n 5, 1996, pp. 595-614.

[11] Lou X. and Loparo K.A., "Bearing fault diagnosis based on wavelet transform and fuzzy inference," Mechanical Systems and Signal Processing, v 18, 2004, pp. 10771095

[12] Michigan Metrology: Surface rougness and wear measurement, 2D Profile parameters in bearing manufacturing, http://www.michmet.com/2d_stylus_parameters_ rkrpkrvk.htm

[13] OpenCV documetion directory: http://docs.opencv.org/_images/ optimal-hyperplane.png

[14] Pokorádi L.: Fuzzy Logic-Based Maintenance Decision. Bulletins in Aeronautical Sciences, XIV. volume 1., 2002., p. 153-158

[15] Purushotham V., Narayanan S., and Prasad S.A.N, "Multi-fault diagnosis of rolling bearing elements using wavelet analysis and hidden Markov model based fault recognition," NDT\&E International , v 38, 2005, pp. 654-664.

[16] Samanta B., Al-Balushi K.R., and Al-Araimi S.A., "Artificial neural network and support vector machines with genetic al gorithm for bearing fault detection," Engineering Applications of artificial Intelligence, v 16, 2003, pp. 657-665.

[17] Schaeffler group: Rolling Bearing Damage, Recognition of damage and bearing inspection, Publ. No. WL 82 102/3 EA, 2001.

[18] Yang J., Zhang Y., and Zhu Y., "Intelligent fault diagnosis of rolling element bearing based on SVMs and fractal dimension," Mechanical Systems and Signal Processing, v 21, n 5, 2007, pp. 2012-2024.

[19] Vachtsevanos G. and Wang P., "Fault prognosis using dynamic wavelet neural networks," IEEE Systems Readiness Technology, 2001, pp. 857-870. 